\title{
Entropy Admissibility of the Limit Solution for a Nonlocal Model of Traffic Flow
}

\author{
Alberto Bressan and Wen Shen \\ Department of Mathematics, Penn State University. \\ University Park, PA 16802, USA. \\ e-mails: axb62@psu.edu, wxs27@psu.edu
}

November 12, 2020

\begin{abstract}
We consider a conservation law model of traffic flow, where the velocity of each car depends on a weighted average of the traffic density $\rho$ ahead. The averaging kernel is of exponential type: $w_{\varepsilon}(s)=\varepsilon^{-1} e^{-s / \varepsilon}$. For any decreasing velocity function $v$, we prove that, as $\varepsilon \rightarrow 0$, the limit of solutions to the nonlocal equation coincides with the unique entropy-admissible solution to the scalar conservation law $\rho_{t}+(\rho v(\rho))_{x}=0$.
\end{abstract}

\section{Introduction}

We consider a nonlocal PDE model for traffic flow, where the traffic density $\rho=\rho(t, x)$ satisfies a scalar conservation law with nonlocal flux

$$
\rho_{t}+(\rho v(q))_{x}=0 .
$$

Here $\rho \mapsto v(\rho)$ is a decreasing function, modeling the velocity of cars depending on the traffic density, while the integral

$$
q(x)=\int_{x}^{+\infty} \varepsilon^{-1} e^{(x-y) / \varepsilon} \rho(y) d s
$$

computes a weighted average of the density of cars ahead. As in [2], we shall assume

(A1) The velocity function $v:\left[0, \rho_{\text {jam }}\right] \mapsto \mathbb{R}_{+}$is $\mathcal{C}^{2}$, and satisfies

$$
v\left(\rho_{\text {jam }}\right)=0, \quad v^{\prime}(\rho) \leq-\delta_{*}<0, \quad \text { for all } \rho \in\left[0, \rho_{\text {jam }}\right] .
$$

One can think of $\rho_{\text {jam }}$ as the maximum possible density of cars along the road, when all cars are packed bumper-to-bumper and nobody moves. The conservation equation (1.1) will be solved with initial data

$$
\rho(0, x)=\bar{\rho}(x) \in\left[0, \rho_{\text {jam }}\right] .
$$


As $\varepsilon \rightarrow 0+$, the weight function $w_{\varepsilon}(s)=\varepsilon^{-1} e^{-s / \varepsilon}$ converges to a Dirac mass at the origin, and the nonlocal equation (1.1)-(1.2) formally converges to the scalar conservation law

$$
\rho_{t}+(\rho v(\rho))_{x}=0 .
$$

Assuming that the initial datum $\bar{\rho}$ has bounded total variation and takes uniformly positive values, the recent analysis in [2] has established:

(i) For every $\varepsilon>0$, the Cauchy problem with non-local flux (1.1), (1.2), (1.4), has a unique solution $\rho=\rho_{\varepsilon}(t, x)$. Its total variation satisfies a uniform bound

$$
\operatorname{Tot.Var} \cdot\left\{\rho_{\varepsilon}(t, \cdot)\right\} \leq M
$$

where the constant $M$ is independent of $t, \varepsilon$.

(ii) As $\varepsilon \rightarrow 0$, by possibly taking a subsequence, one obtains the convergence $\rho_{\varepsilon} \rightarrow \rho$ in $\mathbf{L}_{l o c}^{1}$. The limit function $\rho=\rho(t, x)$ provides a weak solution to the Cauchy problem (1.4)-(1.5).

A major issue, which was not fully resolved in [2], is the entropy admissibility of the limit solution $\rho$. Aim of the present note is to resolve this question in the affirmative. Namely, we prove:

Theorem. Let $v$ satisfy the assumptions (A1), and let $\rho_{\varepsilon}$ be a sequence of solutions to the nonlocal Cauchy problem (1.1), (1.2) and (1.4), satisfying the uniform BV bounds (1.6). Assume that, as $\varepsilon \rightarrow 0$, we have the convergence $\rho_{\varepsilon} \rightarrow \rho$ in $\mathbf{L}_{\text {loc }}^{1}$.

Then $\rho$ is the unique entropy admissible solution to the Cauchy problem (1.4)-(1.5).

The above result was proved in [2] in the special case where the velocity is affine: $v(\rho)=$

$a-b \rho$. The earlier proof was based on the Hardy-Littlewood inequality. In the next section we give a simpler proof, valid for a general class of velocity functions $v$.

For a more general class of averaging kernels, assuming that the initial datum $\bar{\rho}$ satisfies a one-sided Lipschitz condition, the convergence to the unique entropy admissible solution was recently proved in [3]. Our result requires an exponential kernel, but it applies to any BV initial data. In particular, $\bar{\rho}$ can be piecewise constant.

For the general theory of conservation laws we refer to $[1,5,6]$. A brief review of literature on hyperbolic conservation laws with nonlocal flux can be found in [2].

\section{Proof of the theorem}

1. According to $[4,7]$, to prove uniqueness it suffices to prove that the limit solution dissipates one single strictly convex entropy. We thus consider the entropy and entropy flux pair

$$
\eta(\rho)=\frac{\rho^{2}}{2}, \quad \psi(\rho)=\int_{0}^{\rho}\left[s v(s)+s^{2} v^{\prime}(s)\right] d s .
$$


For future use, we observe that (1.2) implies

$$
\rho=q-\varepsilon q_{x}
$$

Moreover, we introduce the function

$$
W(\rho) \doteq \int_{0}^{\rho} s^{2} v^{\prime}(s) d s
$$

The equation (1.1) can now be written as

$$
\rho_{t}+(\rho v(\rho))_{x}=(\rho(v(\rho)-v(q)))_{x} .
$$

Multiplying both sides by $\eta^{\prime}(\rho)=\rho$, we obtain

$$
\eta(\rho)_{t}+\psi(\rho)_{x}=\rho(\rho(v(\rho)-v(q)))_{x} .
$$

2. Given a test function $\varphi \in \mathcal{C}_{c}^{1}(\mathbb{R}), \varphi \geq 0$, using (2.2) we estimate the quantity

$$
\begin{aligned}
J & \doteq 2 \int \rho(\rho(v(\rho)-v(q)))_{x} \varphi d x \\
& =\int\left(\rho^{2}\right)_{x}(v(\rho)-v(q)) \varphi d x+\int 2 \rho^{2}(v(\rho)-v(q))_{x} \varphi d x \\
& =-\int \rho^{2}(v(\rho)-v(q)) \varphi_{x} d s+\int \rho^{2}(v(\rho)-v(q))_{x} \varphi d x \\
& \doteq J_{1}+J_{2} .
\end{aligned}
$$

Concerning the second integral, using (2.2) we obtain

$$
\begin{aligned}
J_{2} & =\int \rho^{2} v^{\prime}(\rho) \rho_{x} \varphi d x-\int \rho q v^{\prime}(q) q_{x} \varphi d x+\int \rho \varepsilon\left(q_{x}\right)^{2} v^{\prime}(q) \varphi d x \\
& \doteq J_{21}+J_{22}+J_{23} .
\end{aligned}
$$

Using (2.2) once again, we now compute

$$
\begin{aligned}
J_{21}+J_{22} & =\int \rho^{2} v^{\prime}(\rho) \rho_{x} \varphi d x-\int q^{2} v^{\prime}(q) q_{x} \varphi d x+\int q \varepsilon\left(q_{x}\right)^{2} v^{\prime}(q) \varphi d x \\
& \doteq J_{3}+J_{4}+J_{5} .
\end{aligned}
$$

Since $\rho, q, \varphi \geq 0$ while $v^{\prime} \leq 0$, from (2.6) and (2.7) we immediately see that

$$
J_{23} \leq 0, \quad J_{5} \leq 0 .
$$

On the other hand, integrating by parts and recalling (2.3), we obtain

$$
\begin{aligned}
J_{3}+J_{4} & =\int[W(\rho)]_{x} \varphi d x-\int[W(q)]_{x} \varphi d x \\
& =-\int[W(\rho)-W(q)] \varphi_{x} d x .
\end{aligned}
$$


3. To conclude, consider a sequence of solutions $\rho_{\varepsilon}$ to (1.1)-(1.2), (1.4). Assume that, as $\varepsilon \rightarrow 0$, we have the convergence $\rho_{\varepsilon} \rightarrow \rho$ in $\mathbf{L}_{l o c}^{1}$. Notice that this implies $q_{\varepsilon} \rightarrow \rho$ in $\mathbf{L}_{l o c}^{1}$ as well. Hence, the integrals $J_{1}$ and $J_{3}+J_{4}$ both approach zero. By the previous analysis,

$$
\begin{aligned}
& 2 \iint\left\{\eta\left(\rho_{\varepsilon}\right) \varphi_{t}+\psi\left(\rho_{\varepsilon}\right) \varphi_{x}\right\} d x d t \\
& \quad \geq \iint \rho_{\varepsilon}^{2}\left(v\left(\rho_{\varepsilon}\right)-v\left(q_{\varepsilon}\right)\right) \varphi_{x} d x d t+\iint\left[W\left(\rho_{\varepsilon}\right)-W\left(q_{\varepsilon}\right)\right] \varphi_{x} d x d t
\end{aligned}
$$

Letting $\varepsilon \rightarrow 0$, since the right hand side converges to zero, we obtain

$$
\iint\left\{\eta(\rho) \varphi_{t}+\psi(\rho) \varphi_{x}\right\} d x d t \geq 0
$$

This proves that the limit solution $\rho$ is entropy admissible. In particular, by $[4,7], \rho$ is the unique entropy weak solution to the Cauchy problem (1.4)-(1.5).

Acknowledgment. This research was partially supported by NSF with grant DMS2006884, "Singularities and error bounds for hyperbolic equations".

\section{References}

[1] A. Bressan, Hyperbolic Systems of Conservation Laws. The One Dimensional Cauchy Problem, Oxford University Press, 2000.

[2] A. Bressan and W. Shen, On traffic flow with nonlocal flux: a relaxation representation, Arch. Rational Mech. Anal. 237 (2020), 1213-1236.

[3] M. Colombo, G. Crippa, E. Marconi, and L.V. Spinolo, Local limit of nonlocal traffic models: convergence results and total variation blow-up. Preprint 2019, arXiv:1808.03529v2.

[4] C. De Lellis, F. Otto, and M. Westdickenberg, Minimal entropy conditions for Burgers equation. Quart. Appl. Math. 62 (2004), 687-700.

[5] C. Dafermos, Hyperbolic Conservation Laws in Continuum Physics, Fourth edition. Springer-Verlag, Berlin, 2016.

[6] H. Holden and N. Risebro, Front Tracking for Hyperbolic Conservation Laws. SpringerVerlag, Berlin, 2002.

[7] E. Y. Panov. Uniqueness of the solution of the Cauchy problem for a first order quasilinear equation with one admissible strictly convex entropy. (Russian) Mat. Zametki 55 (1994), 116-129; translation in Math. Notes 55 (1994), 517-525. 